\title{
Performance Analysis of Rayleigh and Rician Fading Channel Models using Matlab Simulation
}

\author{
Sanjiv Kumar \\ Department of Computer Engineering, BPS Mahila Vishwavidyalaya, Khanpur, Kalan-131305, India \\ E-mail: skganghas@rediffmail.com \\ P. K. Gupta \\ Department of Computer Science and Engineering, \\ Jaypee University of Information Technology, Waknaghat, Solan - 173 234, India \\ E-mail: pradeep1976@yahoo.com \\ G. Singh \\ Department of Electronics and Communication Engineering, \\ Jaypee University of Information Technology, Waknaghat, Solan - 173 234, India \\ E-mail: ghanshyam.singh@juit.ac.in \\ D. S. Chauhan \\ Uttarakhand Technical University, Deharadun, India \\ E-mail:drdschauhan@gmail.com
}

\begin{abstract}
An effort has been made to illustrate the performance comparison of the Rayleigh and Rician fading channel models by using MATLAB simulation in terms of source velocity and outage probability. We have developed algorith ms for the Ray leigh and Rician fading channels, which computes the envelop and outage probability. The parameters such as source velocity and outage probability play very important role in the performance analysis and design of the digital communication systems over the multipath fading environment.
\end{abstract}

Index Terms - Fading Channel, Wireless Communication, Ray leigh Fading, Rician Fading, Error Rate, Outage Probability

\section{Introduction}

An explosive development of the wire less technology has opened several new paths for its implementation, however some unavoidable circumstances attenuate the signal energy and make barriers to achieving the optimum results from the system [1]. The radio link between the transmitter and receiver varies from simple line-of-sight to one that is severely obstructed by the buildings, mountains etc, and hence suffers from severe multipath fading [1-7]. However, the mobile channels are very different from the stationary as well as predictable wired channels, because of their rando mness. There are several factors which determine the behavior of a channel such as terrain features between the transmitter and receiver, the speed of transmitter and receiver, weather conditions etc. Over the years, several studies and measurements have been undertaken in different locations for such channels and various models have been proposed for both the indoor and outdoor environments [3, 4]. The instantaneous signal strength at the receiver can be predicted using the traditional large-scale and small-scale models, where in the largescale models predict the average received signal strength depending on the transmitter-receiver distance and the small-scale channel models represent local variations of the average signal strengths [8-13]. The lognormal model, in which the measured signal levels have a normal distribution about the mean received signal, are suitable for emu lating the large scale fading effects for the mobile channels models. The normal deviation about the mean captures the random shadowing effects that occur because of the variation in the clutter surrounding a moving vehicle, though the mean signal strength at the vehicular receiver remains the same. The frameworks for modeling and simulating the satellite channel along with the time variations have been extensively reported in the literature [14-16]. It is widely accepted that, while the lognormal-Rayleigh model accurately depicts the shadowed channel, a Rician channel model is more appropriate for the unshadowed channel [9-21].

However, the error performance modelling of the wireless channels is inherently depends upon the radio propagation modes such as line-of-sight, reflections, diffraction and scattering caused by an object with 
dimensions on the order of wavelength [1]. One of the most disturbing aspects in the wireless communication is fading, which is present when there are several multipath components and these components arrive at the receiver at slightly different times. If there is movement in the system then there is also phase difference between the received components, which leads to shift in the frequency. However, in the multipath propagation, the movement of the transmitter/receiver or both and bandwidth of the signal are the factors that influence the fading and multipath delay nature of the channel, which is quantified by the delay spread and coherence bandwidth [11]. The timevarying nature of the channel caused by the movement is quantified by Doppler spread and Coherence time [16-21]. In the high mobility scenario, the relative motion between the transmitters and receivers results rapid time variation and significant Doppler shift. Accumulating dynamically changed multipath effects and noise, a significant fluctuation in the received signal strength is observed in the channel. However, the fading is often modelled in the literature with the Rayleigh fading model. The Ray leigh fading model has not been challenged until very recently when researchers started to focus on the throughput problem at vehicular speeds. The Rayleigh fading model works on the assumption that the resultant fading arises from a large number of uncorrelated partial waves with identically distributed amplitudes and uniformly distributed random phases [19-21]. This assumption is highly optimistic in the mobile communication environment at high vehicular speeds. However, more realistic assumption is to have many partial waves with amplitudes which follow the distributions that are not identical, yet are partially correlated $[19,21]$. Doppler spread is another effect of mobility, which changes the signal frequency over time. When the coherence time becomes small relative to the delay constraint of the channel, it results bit error rate at the receiver, which depends on the fading severity of the channel. According to the fading severity, the channel is divided into three categories, and different distribution models are used to estimate that channel according to the type of fading, which is described in the following section.

In this paper, we have developed an algorith $m$ for the Rayleigh and Rician fading channel in a vehicular environment. The remainder of the paper is organized as follows. Section II discusses the performance of the Rayleigh fading model and proposes algorithm. Section III explores the Rician fading channel model and developed an algorithm for it. Finally, Section IV concludes the work.

\section{Performance Analysis of Rayleigh Fading Channel Model}

In a heavily built-up urban or where there is non-lineof-sight communication between the transmitter and receiver, the objects in the environment attenuate, reflect, refract, and diffract the signal before it arrives at the receiver. This propagation environment is known as Rayleigh fading, and the Rayleigh distribution model is a specialized stochastic fading model for this type of fading environment. The channel fading depends on moving speed of the receiver and/or transmitter. The amplitude gain of this fading channel is characterized by the Rayleigh distribution [19]. However, the constructive and destructive nature of the multipath components in the flat fading channels can be approximated by the Rayleigh distribution if there is no line-of-sight, which means when there is no direct path between the transmitter and receiver. The mobile antenna, instead of receiving the signal over one line-ofsight path, receives a number of reflected and scattered waves because of the varying path lengths, the phases are random, and consequently, the instantaneous received power becomes a random variable. In the case of an un-modulated carrier, the trans mitted signal at frequency $\omega_{c}$ reaches the receiver $v$ ia a nu mber of paths, the $\mathrm{i}^{\text {th }}$ path having an amplitude $\mathrm{a}_{\mathrm{i}}$, and a phase $\phi_{i}$. If we assume that there is no direct path or line-of sight component, the received signal s ( $t$ ) can be expressed as [9]:

$$
s(t)=\sum_{i=1}^{N} a_{i} \cos \left(\omega_{c} t+\Phi_{i}\right)
$$

where $N$ is the number of paths. The phase $\phi_{i}$ depends on the varying path lengths and change by 2 when the path length changes by a wavelength. When there is relative motion between the transmitter and the receiver, the Equation (1) can be modified to include the effects of the motion induced frequency and phase shifts.

Let the $i^{\text {th }}$ reflected wave with amplitude $a_{i}$ and phase $\phi_{i}$ arrive at the receiver from an angle $\psi_{i}$ relative to the direction of motion of the antenna. The Doppler shift of this wave is given by:

$$
\omega_{d i}=\frac{\omega_{c} v}{c} \cos \psi_{i}
$$

where $v$ is the velocity of the mobile object, $c$ is the speed of light, and the $\psi_{\mathrm{i}}$ 's are uniformly distributed over $[0,2 \pi]$. The received signal $s(t)$ can now be written as:

$$
s(t)=\sum_{i=1}^{N} a_{i} \cos \left(\omega_{c} t+\omega_{d i} t+\Phi_{i}\right)
$$

By expressing the signal in phase and Quadrate form, Equation (3) can be written as:

$$
s(t)=I(t) \cos \omega_{c} t+Q(t) \sin \omega_{c} t
$$

where the in-phase and Quadrate components are given as: 


$$
\begin{array}{r}
I(t)=\sum_{i=1}^{N} a_{i} \cos \left(\omega_{d i} t+\Phi_{i}\right) \\
Q(t)=\sum_{i=1}^{N} a_{i} \sin \left(\omega_{d i} t+\Phi_{i}\right)
\end{array}
$$

and the envelope $\mathrm{R}$ is given by:

$$
R=\sqrt{[I(t)]^{2}+[Q(t)]^{2}}
$$

When $N$ is large, the in-phase and Quadrate components will be Gaussian nature. The probability density function (PDF) of the received signal envelope, $f(\mathrm{r})$, can be shown to be Rayleigh given by $[12,21]$ :

$$
f(r)=\frac{r}{\sigma^{2}} e^{-\frac{r^{2}}{2 \sigma^{2}}}
$$

where $\sigma^{2}$ is the time-average power of the received signal before the envelope detection.

\subsection{Proposed Algorithm for Rayleigh Channel}

In this section, we have proposed the algorithm for Rayleigh fading channel. The proposed algorithm completes its functioning in four different steps, where step-1 declares about the various used variables in the proposed algorithm and step-2 is associated with the sending of data through Rayleigh channel. In step-3, an envelope is designed for received Rayleigh signal using QAM amplitude demodulation on the received signals. Finally, the step- 4 computes the outage probability using the theoretical and simulated methods .

Algorithm: Rayleigh Fading Channel

STEP 1: Variable Declaration

V: Input Velocity in miles/sec.

P: Input number of paths

T: Time array

1: Carrier frequency

П: Sampling frequency

$\omega:$ Radian frequency

$\delta$ : Data frequency

$\tau$ : Sampling periods

o: Received Rayleigh signals

$\rho:$ Threshold power

$\delta$ : Threshold outage

б: Weibull distributiob function

E: Rayleigh envelop

\section{STEP 2: Sending of Data through Rayleigh Channel}

$\Theta \leftarrow$ Make an array of zeros [T]

FOR $I:=1: P$

random distribution);

$$
\text { Calculates the value of } \delta \text {; }
$$

$\delta \leftarrow 2 * p i * V^{*} \Lambda * \cos ($ for uniform

Find the value of $\sigma$;

$\theta \leftarrow \theta+\sigma^{*} \cos (\Lambda+\eta) * T+$ value of uniform random distribution;

END;

\section{STEP 3: Making Envelop of received Rayleigh Signal}

Performing Quadrature amplitude demodulation for signals;

$$
E \leftarrow \operatorname{sqrt}\left(\theta i^{\wedge} 2+\theta q^{\wedge} 2\right)
$$

\section{STEP 4: Computation of Outage probability}

FOR $X:=1: P$

Calculate the value of $\rho$;

Calculate the value of $\delta$;

count $=0$;

$$
\begin{aligned}
\text { FOR } Y= & 1: \text { length }(E) \\
\text { power }= & \text { power in decibel } ; \\
& \text { IF power }<=\rho \\
& \text { count }++
\end{aligned}
$$

ELSE

END;

END;

Find the simulated outage probability;

\section{END;}

\subsection{Simulation Results for Rayleigh Channel}

Figure 1, shows the RF signal and its envelop plot. As the speed of the user is increased from $0 \mathrm{~m} / \mathrm{s}$ to 50 $\mathrm{m} / \mathrm{s}$, we can see that the amount of fading is increased in the signal envelop. In this simulation, we have set the threshold to 2.3 volt. As we increase the speed, more of the signal goes below the threshold. Hence the fading is one of the major problems in case of wireless communication. 

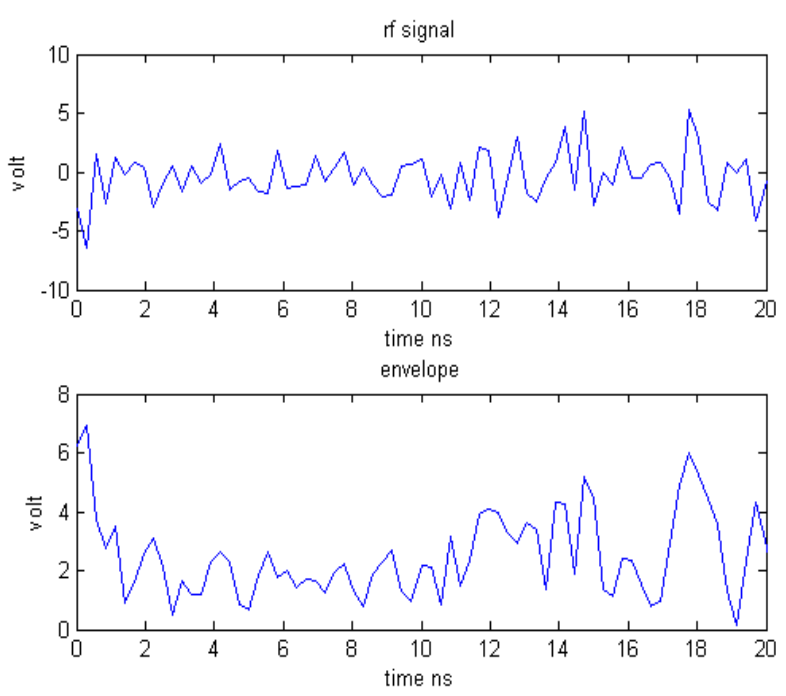

(a)
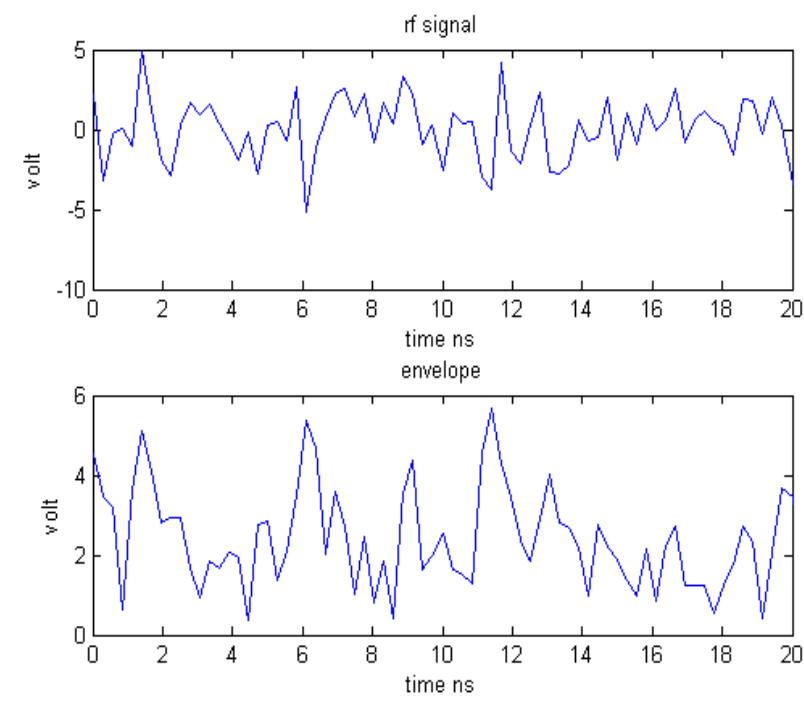

(b)
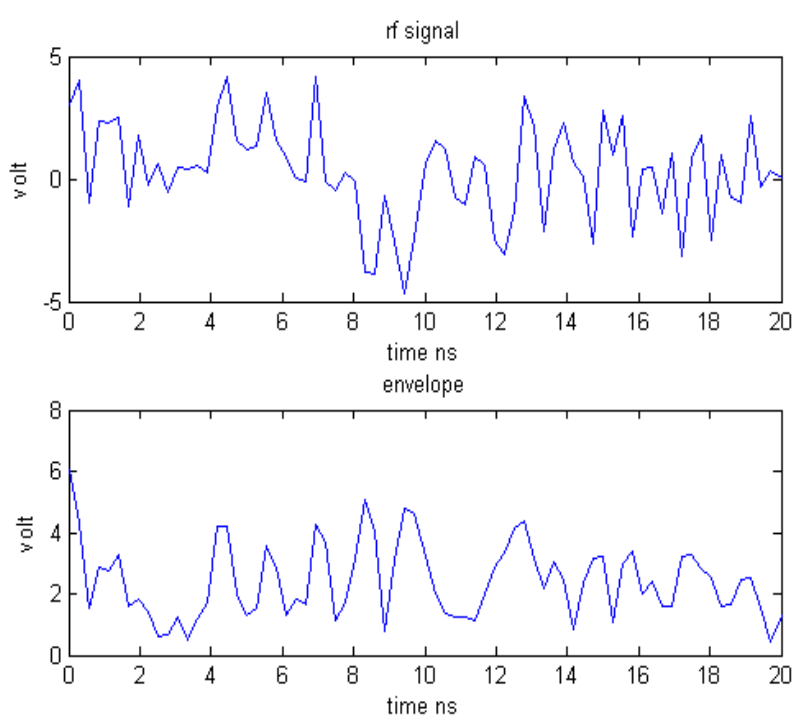

(c)
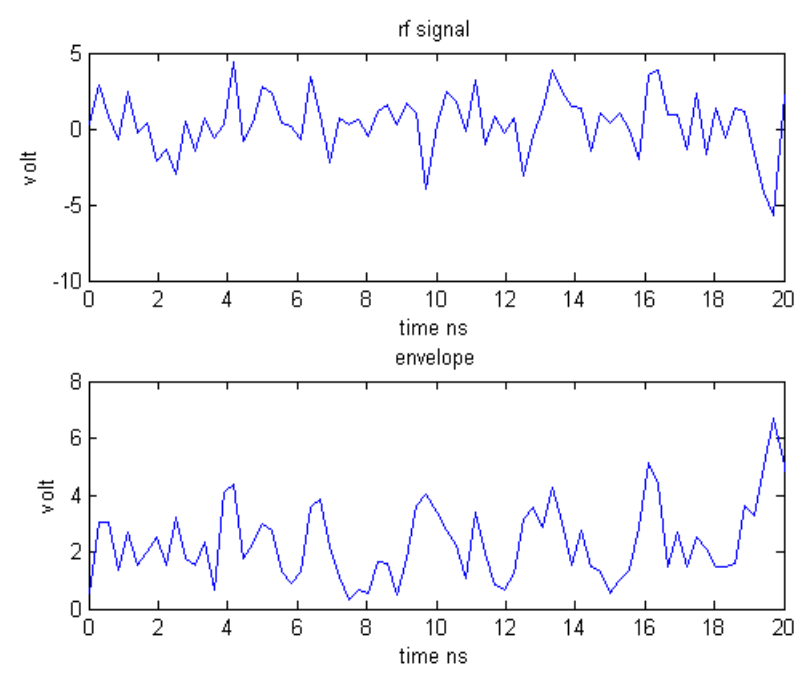

(d)

Fig. 1: Simulated radio frequency signal by using proposed algorithm for Rayleigh fading channel for

(a) stationary source, (b) at source velocity $10 \mathrm{~m} / \mathrm{s}$,

(c) source velocity $25 \mathrm{~m} / \mathrm{s}$, and (d) at source velocity $50 \mathrm{~m} / \mathrm{s}$

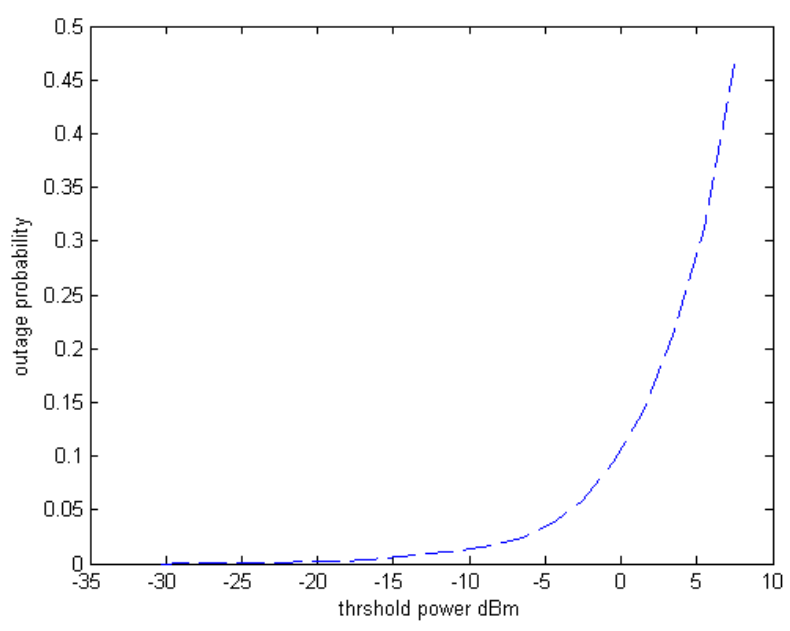

Fig. 2: Outage probabilities for the Rayleigh fading channel

The outage probability is the ratio of the number of samples of the signal below the threshold to the total number of samples. From Fig. 2, it is clear that as we increase the threshold power, the outage probability is increased, that is the probability of better detection of signal is decreased. A key requirement for such resource management schemes is to have an analytical model that can estimate the bit-error-rate and outage probability at high vehicular speeds, so that proactive actions can be taken and proper planning can be done. However, the fading models are used for the bit error rate and outage probability estimation. The fading channel caused by multipath and Doppler shift and often modelled with the popular Rayleigh distribution [21]. The Rayleigh fading model has not been challenged until very recently when researchers started to focus on high throughput at vehicular speeds. The Rayleigh model works on the assumption that the resultant fading arises from a large number of 
uncorrelated partial waves, with identically distributed amplitudes, and uniformly $[0,2 \pi]$ distributed random phases [19,21]. This assumption is highly optimistic in a mobile communication environment at high vehicular speeds.

\section{Rician Fading Channel Model}

When there is line-of-sight, direct path is normally the strongest component goes into deeper fade compared to the multipath components. This kind of signal is approximated by Rician distribution $[4,19,20]$. As the dominating component run into more fade the signal characteristic goes from Rician to Rayleigh distribution $[16,19]$. The derivation of the probability density function of the amplitude is more involved than for Ray leigh fading, and a Bessel function occurs in the mathe matical expression. In the presence of such a path, the transmitted signal can be written as:

$$
\begin{aligned}
s(t)= & \sum_{i=1}^{N-1} a_{i} \cos \left(\omega_{c} t+\omega_{d i} t+\varphi_{i}\right) \\
& +k_{d} \cos \left(\omega_{c} t+\omega_{d} t\right)
\end{aligned}
$$

where the constant $k_{d}$ is the strength of the direct component, $\omega_{\mathrm{d}}$ is the Doppler shift along the line-ofsight path, and $\omega_{\mathrm{di}}$ are the Doppler shifts along the indirect paths. The envelope in this case has a Rician density function given by $[4,11]$ :

$$
f(r)=\frac{r}{\sigma^{2}} e^{-\left\{\frac{r^{2}+k_{d}^{2}}{2 \sigma^{2}}\right\} \cdot I_{0}\left\{\frac{r k_{d}}{\sigma^{2}}\right\}}
$$

where $I_{0}(0)$ the $0^{\text {th }}$ order is modified Bessel function of the first kind

\subsection{Proposed Algorithm for Rician Channel}

In this section, we have developed an algorithm for the Rician fading channel. The proposed algorithm completes its functioning in four different steps where step-1 declares about the various used variables in the proposed algorithm and step-2 is associated with the sending of data through the Rayleigh fading channel. In step-3 an envelope is designed for received Rayleigh signal using QAM amplitude demodulation on received signals. Finally, the step-4 calculates the outage probability using theoretical and simulated methods

\begin{tabular}{l}
\hline Algorithm: Rician Fading Channel \\
\hline STEP 1: Variable Declaration \\
V: Input Velocity in miles/sec. \\
P: Input number of paths
\end{tabular}
T: Time array
1: Carrier frequency
D: Data frequency
П: Sampling frequency

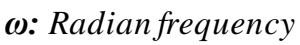

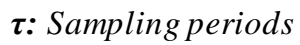
$\boldsymbol{\theta}:$ Received Rician signals
$\rho:$ Threshold power

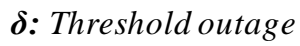
$\sigma:$ Weibull distribution function
$\boldsymbol{E}:$ Rician envelop

STEP 2: Sending of Data through Rayleigh Channel

$\theta \leftarrow$ Make an array of zeros [T]

FOR $I:=1: P$

random distribution);

Calculates the value of $D$;

$D \leftarrow 2 * p i * V^{*} \Lambda * \cos ($ for uniform

Find the value of $\sigma$;

$\theta \leftarrow \theta+\sigma^{*} \cos (\Lambda+\eta) * T+$ value of

uniform random distribution;

END;

\section{STEP 3: Making Envelop of received Rayleigh} Signal

Performing Quadrature amplitude demodulation for signals;

$$
E \leftarrow \operatorname{sqrt}\left(\theta i^{\wedge} 2+\theta q^{\wedge} 2\right)
$$

\section{STEP 4: Computation of Outage probability}

FOR $X:=1: P$

Calculate the value of $\rho$;

Calculate the value of $\delta$;

count $=0$;

$$
\begin{aligned}
\text { FOR } Y= & 1: \text { length }(E) \\
\text { power }= & \text { power in decibel; } \\
& \text { IF power }<=\rho \\
& \text { count }++
\end{aligned}
$$

ELSE

END; 
Find the simulated outage probability;

END;

\subsection{Simulation Results for Rician Channel}

Figure 3, illustrate the RF signal and its envelop plot for Rician fading channel. As the speed of the user is increased from $0 \mathrm{~m} / \mathrm{s}$ to $50 \mathrm{~m} / \mathrm{s}$, we can see that the amount of the fading is increased in the signal envelop. However, the threshold has been set to 2.3 volt. As we increase the speed, more of the signal goes below the threshold. Hence the fading is one of the major problems in case of wireless communication.
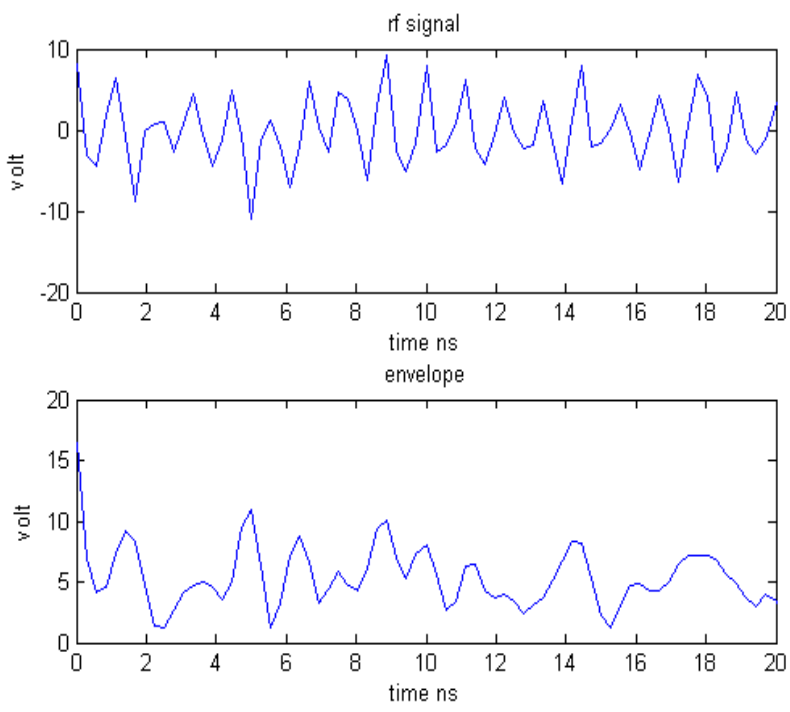

(a)
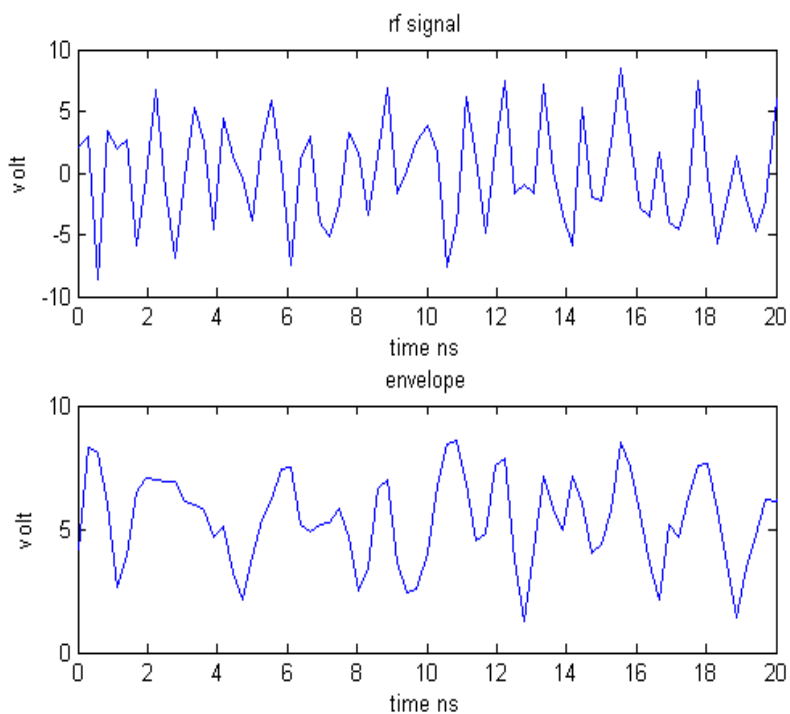

(b)
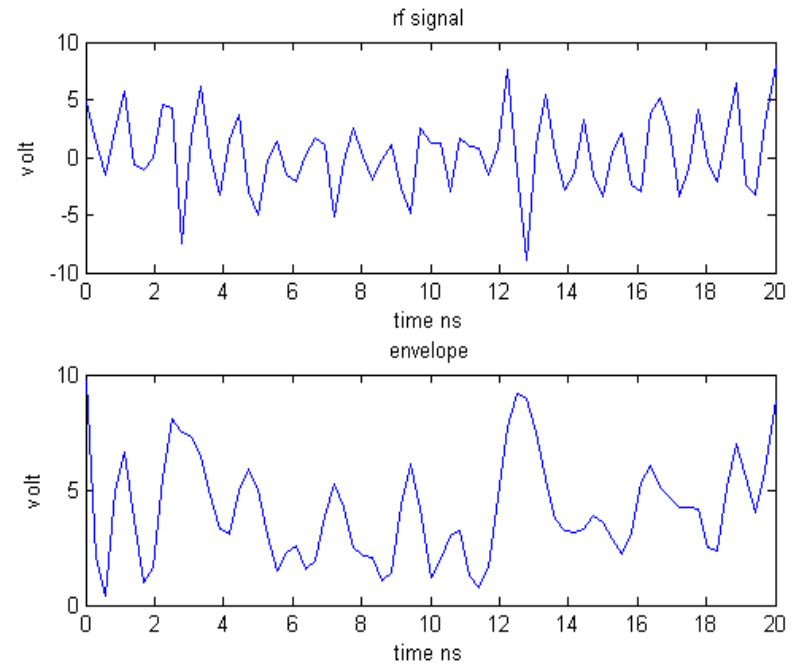

(c)
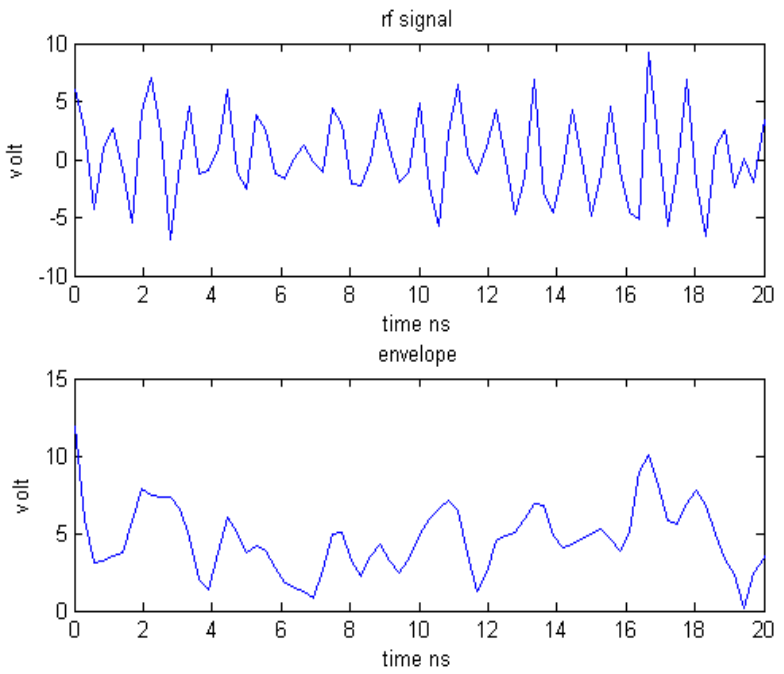

(d)

Fig. 3: Simulated radio frequency signal using proposed algorithm for Rician fading channel for

(a) stationary source, (b) at source velocity $10 \mathrm{~m} / \mathrm{s}$,

(c) source velocity $25 \mathrm{~m} / \mathrm{s}$, and (d) at source velocity $50 \mathrm{~m} / \mathrm{s}$

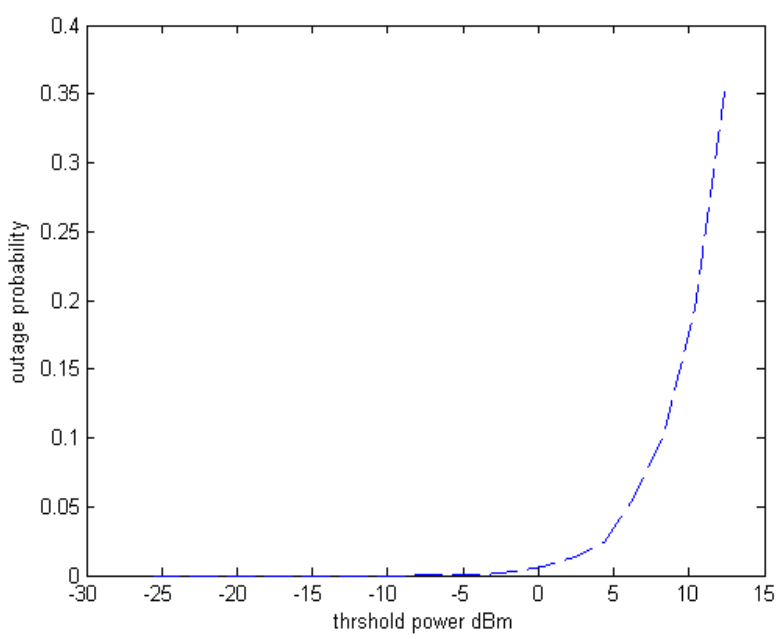

Fig. 4: The outage probabilities for Rician fading channel 
The outage probability is the ratio of the no of samples of the signal below threshold to the total number of samples. From Fig. 4 it is clear that as we increase the threshold power outage probability is increased, that is probability of better detection of signal is decreased. In TABLE 1, the performance of the Rayleigh and Rician fading channel model has been compared. However in both cases (Rayleigh and Rician fading model) the outage probability increases with the increase of the velocity of the moving object.

Table 1: Performance comparison with reference to the outage probability of Rayleigh and Rician fading channels

\begin{tabular}{|c|c|c|}
\hline Mobile velocity (in m/s) & Outage Probability (Rayleigh) & Outage Probability (Rician) \\
\hline 0 & 0.19149 & 0.09311 \\
\hline 2 & 0.19193 & 0.09312 \\
\hline 4 & 0.19246 & 0.09314 \\
\hline 6 & 0.19303 & 0.09346 \\
\hline 8 & 0.19339 & 0.09350 \\
\hline
\end{tabular}

\section{Conclusion}

In this paper, we have simulated the Rayleigh and Rician fading channel models in vehicular environment for the outage probability. From the simulation results, we conclude that as the vehicle speed of user is increased, the amount of fading is increased in the signal envelope. Therefore, as we increase the speed, more of the signal goes below the threshold and the amount of fading increased. We also simulate the Rayleigh and Rician fading channels in terms of the outage probability by using Matlab simulation for different thresholds of the received signal and compare these values to those calculated analytically. However, the results from both the simulation and analysis are comparable. As we have observed from the comparison table, the outage probability in the Rician fading channel is lower than that of the Rayleigh fading channel, which is due to the presence of line-of-sight path in the Rician channel. We also concluded that the outage probability increases as the mobile velocity or Doppler shift increases. Dynamically changed multipath and Doppler effects are the main causes behind the degradation of the channel capacity. However, we would like to develop a generic model for fading in mobile communication (vehicular applications) system, which will be reported in future communication.

\section{Acknowledgements}

The authors are very much thankful to the potential reviewers for their critical comments and suggestions to improve the quality of the manuscript.

\section{References}

[1] Bernard Sklar. Rayleigh Fading Channels in Mobile Digital Communication Systems Part 1: Characterization. IEEE Comm., 1997, v35, n7, pp.90-100.
[2] William C. Jakes. Microwave Mobile Communications. John Wiley, New York, 1974.

[3] John G. Proakis. Digital Communications. McGraw-Hill, Singapore, 1995.

[4] T. S. Rappaport. Wireless Communications Principles and Practice. Prentice Hall, New Jersy, 1999.

[5] R. H. Clarke. A statistical theory of mobile radio reception. Bell Systems Technical Journal, 1968, v47, n6, 1968, pp.957-1000.

[6] P. Dent, G. E. Bottomley, T. Croft. Jakes fading model revisited. Electronics Letters, v29, n13, 1993, pp.1162-1163.

[7] 'Fading' - Online Article in Wikipedia en.wikipedia.org/wiki/Fading

[8] W. R. Bennett. Distribution of the sum of randomly phased components. Quart. Appl. Math, v5, 1948, pp.385-393.

[9] L. Rayleigh. On the resultant of a large number of vibrations of the same pitch and of arbitrary phase. Philosophical Mag., v27, n6, 1880, pp.460-469.

[10] Haowei Bai, Mohammed Antiquzzaman. Error modeling schemes for fading channels in wireless communications. IEEE Comm. Surveys and Tutorials, v5, n2, 2003, pp.2-9.

[11] Matthias Patzold. Mobile Fading Channels. John Wiley, 2002, England.

[12] M. J. Omidi, S. Pasupathy, P. G. Gulak. Joint data and channel estimation for Rayleigh fading channels. Wireless Personal Communications, v10, 1999, pp.319-339.

[13] M. Nakagami, W. C. Hoffman. The $m$-d istribution A General Formula of Intensity Distribution of Fading. Statistical Methods in Radio Wave Propagation, 1960, Pergamon. 
[14] M. Patzold, U. Killat, F. Laue. On the statistical properties of determin istic simulation models for mobile fading channels. IEEE Trans. Vehicular Technology, v47, n1, 1998, pp.254-269.

[15] W. C. Y. Lee. Mobile Communications Design Fundamentals. John Wiley, New York, 1993.

[16] H. Suzuki. A statistical model for urban radio propagation. IEEE Trans. on Comm.,v 27, 1977, pp.673-680.

[17] W. R. Braun, U. Dersch. A physical mobile radio channel model. IEEE Trans. on Vehicular Technology, v40, 1991, pp.472-482.

[18] T. Eng, N. Kong, L. B. Milstein. Comparison of diversity combining techniques for Rayleigh fading channels. IEEE Trans. on Communications, v44, n9, 1996, pp.1117-1129.

[19] C Xiao, Y. Zheng, N C Beaulieu. Novel sum-ofsinusoids simulation model for Rayleigh and Rician fading channels. IEEE Trans. Wireless Communication, v5, n12, 2006, pp.3667-3679.

[20] Y.-D Yao, A U H Sheikh. Outage probability analysis for microcell mobile radio systems with co-channel interferers in Rician/Rayleigh fading environment. Electronic Letter, v26, n13, 1990, pp.864-866.

[21] C. Xiao, Y. Zheng, N. C. Beaulieu. Statistical simulation model for Rayleigh and rician fading. Proceedings of IEEE International Conference on Communication, 2003, pp.3524 3529.

\section{Authors' Profiles}

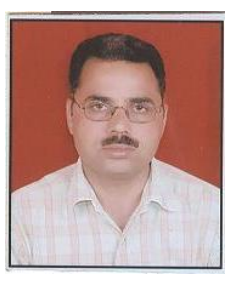

Sanjiv Kumar was born in Kurukshetra, in 1975. He received his B. E. in Electronics and Communication Engineering from the Institution of Engineers (India), Calcutta and M.E. in Electronics and Communication Engineering from Maharishi Dayanand University, Rohtak (Haryana). He is working as a lecturer in the Department of Computer Engineering at B. P. S. Mahila Vishwavidyalaya, Khanpur Kalan (Haryana), India. Currently, he is pursuing his Ph.D. from Uttarakhand Technical University, Dehradun. His research interests include wireless communication, fading channel models.

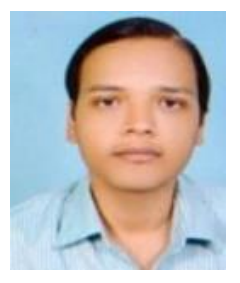

P. K. Gupta received Ph.D. degree in Computer science and Engineering from the Jaypee University of Information Technology, Waknaghat, Solan, India in 2012. He graduated in Informatics and Computer Engineering from Vladimir State University, Vladimir, Russia, in 1999 and received his
M.E. degree in Informatics and Computer Engineering in 2001 from the same university. He has been associated with academics more than ten years in different institutions like BIT M.Nagar, RKGIT Ghaziabad in India. Currently, he is working as Assistant Professor with the Department of Computer Science and Engineering \& ICT, Jaypee University of Information Technology, Waknaghat, Solan, India. He has supervised a number of B.Tech/M.Tech/M.Phil. theses from various universities of India. His research interests include Storage Networks, Green Computing, Software Testing and Cloud Computing. He is a Member of IEEE, Life Member of CSI and Life member of Indian Science Congress As sociation.

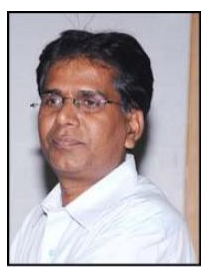

G. Singh: received $\mathrm{Ph} \mathrm{D}$ degree in electronics engineering from the Institute of Technology, Banaras Hindu University, Varanasi, India, in 2000. $\mathrm{He}$ was associated with Central Electronics Engineering Research Institute, Pilani, and Institute for Plas ma Research, Gandhinagar, India, respectively, where he was Research Scientist. He was also worked as an Assistant Professor at Electronics and Communication Engineering Department, Nirma University of Science and Technology, Ahmedanad, India. He was a Visiting Researcher at the Seoul National University, Seoul, S. Korea. At present, he is Associate Professor with the Department of Electronics and Communication Engineering, Jaypee University of Information Technology, Wakanaghat, Solan, India. He is an author and co-author of more than 170 scientific papers of the refereed Journal and International/National Conferences. His research interests include relativistic electronics, surfaceplasmons, Electromagnetics and its applications, nanophotonics, microwave $/ \mathrm{THz}$ antennas and its potential applications.

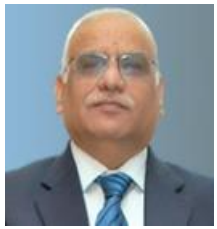

Prof. D. S. Chauhan was born in 1949 at Dholpur, Rajasthan and his parents belong to Mainpuri, Uttar Pradesh. His education took place in Rajasthan, Madhya Pradesh, Uttar Pradesh, Tamilnadu and New Delhi. He did his post doctoral work at Goddard Space Flight Centre, Greenbelf Mary land. USA (1988- 91). He did his B.Sc Engg.(1972) in electrical engineering at I.T. B.H.U., M.E. (1978) at R.E.C. Tiruchirapalli (Madras University) and Ph.D. (1986) at IIT/Delhi. His brilliant career brought him to teaching profession at Banaras Hindu University where he was Lecturer, Reader and then has been Professor till today. He has been director KNIT sultanpur in 1999-2000 and founder vice Chancellor of U.P.Tech. University (2000- 2003-2006). Later on, he has served as Vice-Chancellor of Lovely Profession University (2006-07) and Jaypee University 
of Information Technology (2007-2009) Currently he has been serving as Vice- Chancellor of Uttarakhand Technical University for (2009-till) Tenure. He has been member, NBA-executive AICTE, (2001-04)NABL-DST executive (2002-05) and member, National expert Committee for IIT-NIT research grants.He was Member, University Grant Commission (2006-09). He has been member, CAPART, National executive and chairman central zone, Lucknow from (2001-2004). He has been nominated by UGC as chairman of Advisory committees of four medical universities. Dr Chauhan got best Engineer honour of institution of Engineer in 2001 at Lucknow. He has supervised $24 \mathrm{Ph}$.D., one D.Sc and still guiding number of research scholars. He has authored two books and published and presented 150 research papers in international journals and international conferences.

How to cite this paper: Sanjiv Kumar, P. K. Gupta, G. Singh, D. S. Chauhan,"Performance Analysis of Rayleigh and Rician Fading Channel Models using Matlab Simulation", International Journal of Intelligent Systems and Applications(IJISA), vol.5, no.9, pp.94-102, 2013. DOI: 10.5815/ijisa.2013.09.11 